\title{
M-ary Double-layer Optical Camera Communication For Intelligent Transport System
}

\author{
Xiangdong LI, Luokun LIU \\ Institute of Zhengzhou Information Science and Technology, Zhengzhou, 450001,China. \\ email:Ixd0520@foxmail.com
}

\begin{abstract}
Keywords: optical camera communication (OCC); intelligent transport system (ITS); information superimposition; maximum likelihood (ML) detection.
\end{abstract}

\begin{abstract}
An M-ary double-layer optical camera communication (OCC) system for intelligent transport system (ITS) is proposed. According to the importance of information, the message is split into different priorities. The low-priority data is transmitted with high data rate at the upper layer in high multiplexing mode, whereas the high-priority data is transmitted with high robustness at the lower layer using high diversity scheme. In order to fully utilize the resources of the lower layer which usually occupies more power, the M-ary modulation is introduced into the lower layer signal. Combined with system model, the message can be detected by using a low-complexity ML detection algorithm. Finally, computer simulations verified the system feasibility. With reliable and low-complexity detection, the data rate of the lower layer obtained exponentially enhancement.
\end{abstract}

\section{Introduction}

Visible light communications (VLC) have attracted more and more extensive attention due to its high speed, ubiquitous, green and other advantages [1]. VLC systems usually use light emitting diode (LED) as the sender, and photo detector (PD) to receive with intensity modulation/direct detection. In recent years, with the popularity of handheld (mobile) terminals, the concepts of using the camera as the receiver to communicate with the terminals was put forward and developed rapidly. Since the message is detected and processed from each frame image received by camera, it is also known as optical camera communications (OCC) [2]. Currently OCC has a wide range of applications in the fields of intelligent transportation [3], indoor accurate positioning [4] and invisible information transmission [5].

Recently, many fundamental studies have focused on analyzing system channel capacity [6], studying communication factors [7], constructing an appropriate system model and establishing some experimental prototypes under different scenarios [8]. Double-layer (overlay coding) OCC system was proposed by Japanese scholars, applied in intelligent transport system (ITS). According to the importance of information, the message is divided into different priorities. The high-priority data is loaded on the lower layer using high diversity scheme, suitable for long distance transmission with high robustness but low data rate. On the contrary, the low-priority data is transmitted at the upper layer in high multiplexing mode, applicable to nearly transmission with high data rate but low robustness. Using a low complexity detection algorithm, it can basically realize the extraction of transmitted information in [9]. Through improving the coding, the performance of detection obtained a certain degree of ascension [10].

Based on further optimizing the model of double-layer OCC system, [11] proposed a low complexity detection algorithm based on ML detection, to enhance the performance greatly than sum detection. At the same time, it put forward several signal power allocation schemes for different scenes. Since the lower layer signal is typically a single value loaded on all transmission units in the double-layer OCC system, it is usually assigned to higher transmission power owing to multiplexing gain under certain constraint condition of total power. In order to take full advantage of the limited transmission resources, this article presents using M-ary modulation, for example, quaternary 
modulation to the lower layer signal. With reliable and low-complexity detection, the double-layer OCC system can be achieved stably.

\section{System Overview}

Because the transmitter is usually selected from the LED array or liquid crystal display (LCD), we generally regard portions of the adjacent LEDs or clusters of pixels in LCDs as the transmitting units. The signal on these units is parallel transmitted in the VLC channel after intensity and space modulation. In the receiver image sensors such as high definition (HD) cameras can get image information from transmitter. We can detect and recover transmitted data through post-processing continuous received images.

In the transmitter, the signal loaded on each transmitting unit is actually the superposition of the upper layer and lower layer. Now there are $M \times N$ transmitting units in the sending array. Then the superposed signal on the $(m, n)$ unit at the $k$-th time block is

$$
x_{m, n}[k]=A[k]+B_{m, n}[k], \quad m=1,2, \ldots, M ; n=1,2, \ldots, N
$$

where $A[k]$ is the lower layer quaternary signal on the $(m, n)$ transmitting unit. To compare with the current binary system conveniently, $A[k] \in\left\{-\alpha,-\frac{\alpha}{3},+\frac{\alpha}{3},+\alpha\right\}$, corresponding symbols $\{00,01,10,11\}$ respectively. At the same time, $B_{m, n}[k] \in\{-\beta,+\beta\}$ is on-off keying (OOK) modulation, representing the upper layer signal on this unit.

The signal $x_{m, n}[k]$ could be positive or negative after adding $A[k]$ and $B_{m, n}[k]$. As the driving signal of transmitting units is positive due to intensity modulation, we have to map the superposed signal to be nonnegative. It usually could be realized by adding a direct current signal related to the maximum value of the original.

Due to the HD cameras can achieve the perfect focus within a certain distance (without interference from each unit), this paper assumes that the receiver is accurately received. At the $k$-th time block, the received signal $y_{m, n}^{\prime}[k]$ transmitted from the $(m, n)$ unit is nonnegative because receiving by camera is similar to intensity detection. To post-process conveniently, the signal needs to recover to be bipolar, denoted by $y_{m, n}[k]$. Meanwhile, we can get the $Q$-dimensional column vector $\mathbf{y}[k]$ ( $Q=M \times N)$ by converting the $M \times N$-dimensional received signal matrix $\mathbf{Y}[k]$ for deduction simply. The transformation relationship is not unique. Here is the most common sequence transformation $y_{q}[k]=y_{m, n}[k]$, where $q=(m-1) \times N+n$.

Therefore, the received signals after bipolar and dimension mapping can be expressed as

$$
\mathbf{y}[k]=A[k] \mathbf{e}+\mathbf{B}[k]+\mathbf{n}[k]
$$

where $A[k]$ is the common lower layer signal on all the transmitting units, $\mathbf{e}=[1,1, \ldots, 1]^{T}$ is a $Q$-dimensional column unit vector, $\mathbf{B}=\left[B_{1}, B_{2}, \ldots, B_{Q}\right]^{T}$ denotes the $Q$-dimensional upper layer vector with $B_{q}[k] \in\{-\beta, \beta\}$, noise vector $\mathbf{n}=\left[n_{1}, n_{2}, \ldots, n_{Q}\right]^{T}$ can be modeled as an additive white Gaussian noise[12].

\section{Detection}

At present, detection of binary double-layer OCC system mainly has two algorithms, the sum detection and the ML detection. In [11] the comparisons of performance of two algorithms show that, under the white Gaussian noise channel model, the ML detection algorithm has higher detection performance than the sum detection. Therefore, we adopt ML detection for this system. In the M-ary double-layer OCC system, the objective function for optimization is

$$
\{\hat{A}[k], \hat{\mathbf{B}}[k]\}=\arg \min _{A \in\left\{-\alpha,-\frac{\alpha}{3},+\frac{\alpha}{3},+\alpha\right\}, \mathbf{B}[k] \in\{\beta,-\beta\}^{Q}}\|\mathbf{y}[k]-A \mathbf{e}-\mathbf{B}[k]\|^{2}
$$


If it uses the exhaustive search to detect directly, the complexity of the algorithm is awful high. Think of $A[k]$ is the common lower layer signal on all the transmitting units, we can reduce the complexity of ML detection. If we let

$$
\begin{array}{ll}
\mathbf{y}_{+\boldsymbol{\alpha}}=\mathbf{y}-\alpha \mathbf{e}, & \mathbf{y}_{+\frac{\alpha}{3}}=\mathbf{y}-\frac{\alpha}{3} \mathbf{e}, \\
\mathbf{y}_{-\frac{\boldsymbol{\alpha}}{3}}=\mathbf{y}+\frac{\alpha}{3} \mathbf{e}, & \mathbf{y}_{-\boldsymbol{\alpha}}=\mathbf{y}+\alpha \mathbf{e}
\end{array}
$$

Then, the optimization problem in (3) is equivalent to

$$
\begin{array}{r}
\min \left\{\min _{\mathbf{B}[k] \in\{\beta,-\beta\}^{Q}}\left\|\mathbf{y}_{+\alpha}[k]-\mathbf{B}[k]\right\|^{2}, \min _{\mathbf{B}[k] \in\{\beta,-\beta\}^{Q}}\left\|\mathbf{y}_{+\frac{\alpha}{3}}[k]-\mathbf{B}[k]\right\|^{2},\right. \\
\left.\min _{\mathbf{B}[k] \in\{\beta,-\beta\}^{Q}}\left\|\mathbf{y}_{-\frac{\alpha}{3}}[k]-\mathbf{B}[k]\right\|^{2}, \min _{\mathbf{B}[k] \in\{\beta,-\beta\}^{Q}}\left\|\mathbf{y}_{-\alpha}[k]-\mathbf{B}[k]\right\|^{2}\right\}
\end{array}
$$

The question is to find $\{\hat{A}[k], \hat{\mathbf{B}}[k]\}$ corresponding to the minimum value of the four minimum Euclidean distances in (5). Obviously, when $\mathbf{B}[k]$ has the same sign with $\mathbf{y}_{A}[k], \mathbf{B}[k]=\beta \operatorname{sign}\left(\mathbf{y}_{A}\right)$, each minimum Euclidean distances can achieve a minimum in (5). To search the most minimum, these four minimum Euclidean distances are denoted by variables $z_{1}, z_{2}, z_{3}, z_{4}$ respectively. After comparing the two variables, the smaller is then compared with the next one, until the smallest variable is determined.

The comparison of $z_{1}, z_{2}$ is carried out here as an example, and the comparison between the other variables can expand similarly. Defining the comparison variable $z_{12}=z_{1}-z_{2}$, and combined with simplified scheme in [11], after dividing the real line, which is the range of each $y_{q}[k]$, to three intervals: $\mathrm{I}=\left(-\infty, \frac{\alpha}{3}\right], \mathrm{II}=\left(\frac{\alpha}{3},+\alpha\right]$ and III $=(\alpha,+\infty)$, we can get a low-complexity method

$$
\begin{aligned}
z_{12}= & \sum_{y_{q}[k] \in \mathrm{I}}\left(-\frac{4}{3} \alpha y_{q}\right)+\sum_{y_{q}[k] \in \mathrm{I}}\left(\frac{8}{9} \alpha^{2}-\frac{4}{3} \alpha \beta\right)+\sum_{y_{q}[k] \in \mathrm{II}} 4\left(\beta-\frac{\alpha}{3}\right) y_{q}+ \\
& \sum_{y_{q}[k] \in \mathrm{II}}\left(\frac{8}{9} \alpha^{2}-\frac{8}{3} \alpha \beta\right)+\sum_{y_{q}[k] \in \mathrm{III}}\left(-\frac{4}{3} \alpha y_{q}\right)+\sum_{y_{q}[k] \in \mathrm{III}}\left(\frac{8}{9} \alpha^{2}+\frac{4}{3} \alpha \beta\right)
\end{aligned}
$$

By the sign of $z_{12}$, we can get the smaller between $z_{1}$ and $z_{2}$. Similarly, we can derive other comparison variables, followed by the comparison algorithm for the next step to determine. Since the computational complexity of the algorithm is mainly reflected in the calculation of comparison variables, specifically in the realization of the comparison algorithm, the classification comparison method of the first decision and then calculation can be used to simplify the computational complexity of the algorithm.

Comprehensive above derivation and comparison algorithm, the joint detection of the M-ary lower layer common signal $A[k]$ and the upper layer signal $B_{m, n}[k]$ loaded on each transmission unit can be realized. Finally, $\hat{A}[k]$ is corresponding with $A[k]$ in $z_{\min }$ then, $\hat{\mathbf{B}}[k]=\beta \operatorname{sign}\left(\mathbf{y}_{\hat{A}}\right)$.

\section{Simulation And Analysis}

At the transmitter, in order to adapt to different distribution schemes, we need to distribute power of the signal for each layer, under the normalized processing of the total power of each transmission unit.

$$
\alpha^{2}+\beta^{2}=1
$$

Considering the multiplexing gain of each layer, the power of the lower and upper layer signals are denoted by $E_{A}, E_{B}$, where $E_{A}=Q \alpha^{2}, E_{B}=\beta^{2}$. Obviously, the ratio of $\alpha, \beta$ and the size of $Q$ will ultimately affect the power distribution of each layer signal. In the practical determination of the power allocation scheme, considering the better detection performance, it also needs to take into 
account that the different requirements of each layer for robustness and data rate in the practical application.

Since it is applied to the intelligent transportation system, the lower layer signal should be allocated to higher transmission power for the transmission of important information. In this paper, we set $\alpha: \beta=2: 1$ as the initial condition. We can get the concrete value of $\alpha, \beta$ from (7). Thus, the power ratio of each symbol message in the double layer signal is $\frac{E_{A}}{E_{B}}=4 Q$. With increasing value of $Q$, power assigned to the lower signal is gradually increased, and the gap to the upper signal power increases meanwhile. Table 1 shows the design for signal distribution and the corresponding power and rate information under different transmitting units in this power allocation, assuming the frame rate for 500fps. Since the lower layer is M-ary modulation, when calculating data rate still needs to consider the number of bits per symbol.

Table 1 Power and rate information of each layer under different transmitting units

\begin{tabular}{|c|c|c|c|c|c|c|}
\hline & \multicolumn{2}{|c|}{$Q=4$} & \multicolumn{2}{|c|}{$Q=16$} & \multicolumn{2}{|c|}{$Q=100$} \\
\hline & lower & upper & lower & upper & lower & upper \\
\hline priority & high & low & high & low & high & low \\
\hline number of units per symbol & 4 & 1 & 16 & 1 & 100 & 1 \\
\hline number of symbols per layer & 1 & 4 & 1 & 16 & 1 & 100 \\
\hline power of per symbol & 3.2 & 0.2 & 12.8 & 0.2 & 80 & 0.2 \\
\hline data rate (frame rate $500 \mathrm{fps}$ ) & $1 \mathrm{kbps}$ & 2kbps & $1 \mathrm{kbps}$ & 8kbps & $1 \mathrm{kbps}$ & 50kbps \\
\hline
\end{tabular}

In condition of $Q=16$, the overall performance of the system is investigated under different modulation and detection algorithm schemes. Fig. 1 shows that using the block error rate (identifying the data blocks wrong if arbitrary layer data occur misjudgment) to measure the overall performance of the double-layer OCC system, although the performance of quaternary ML detection scheme is slightly worse compared with binary ML detection, but it can still achieve the block error rate performance of $10^{-5}$ when the signal-to noise ratio (SNR) is $17 \mathrm{~dB}$ around, while the data rate of the lower layer obtains exponentially enhancement. In addition, the M-ary ML detection scheme outperforms the binary summation detection system.

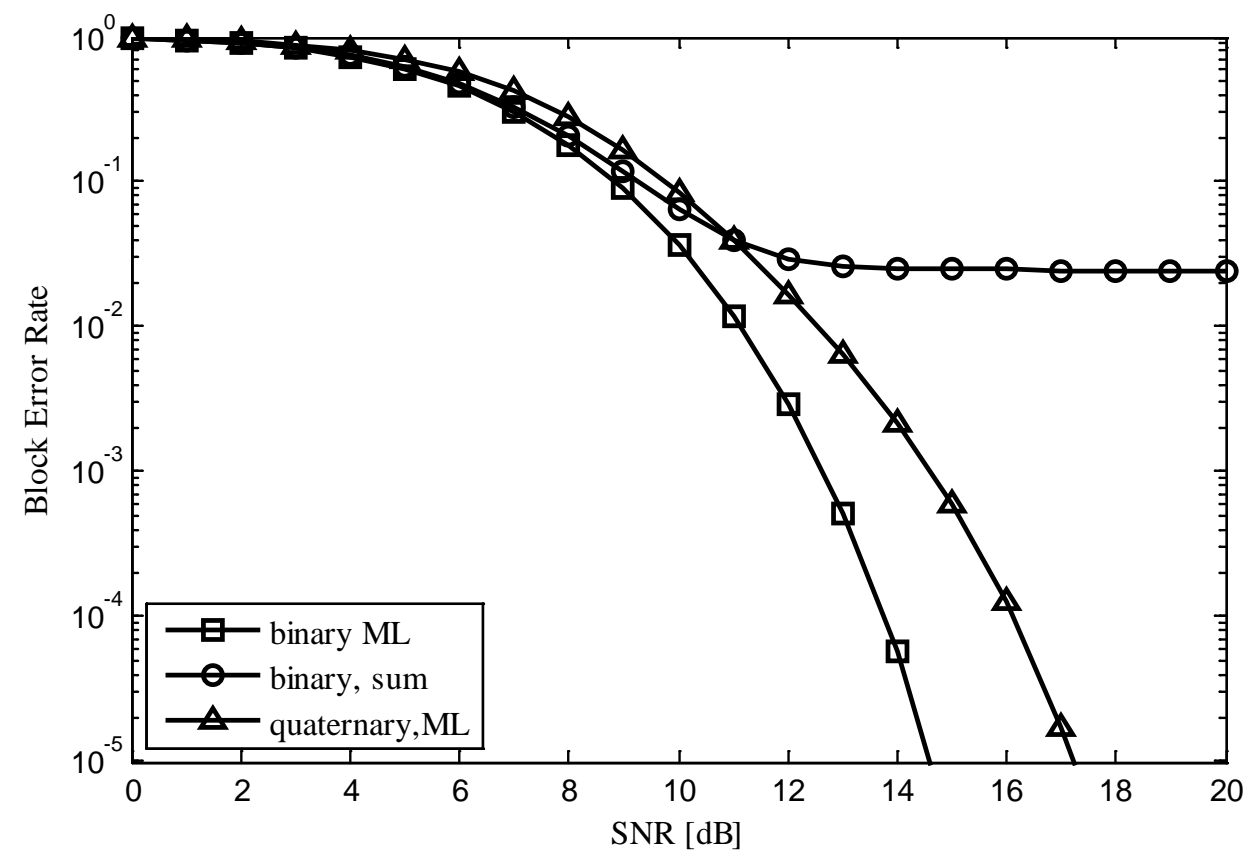

Fig.1 Overall performance under diffient modulation and detection 


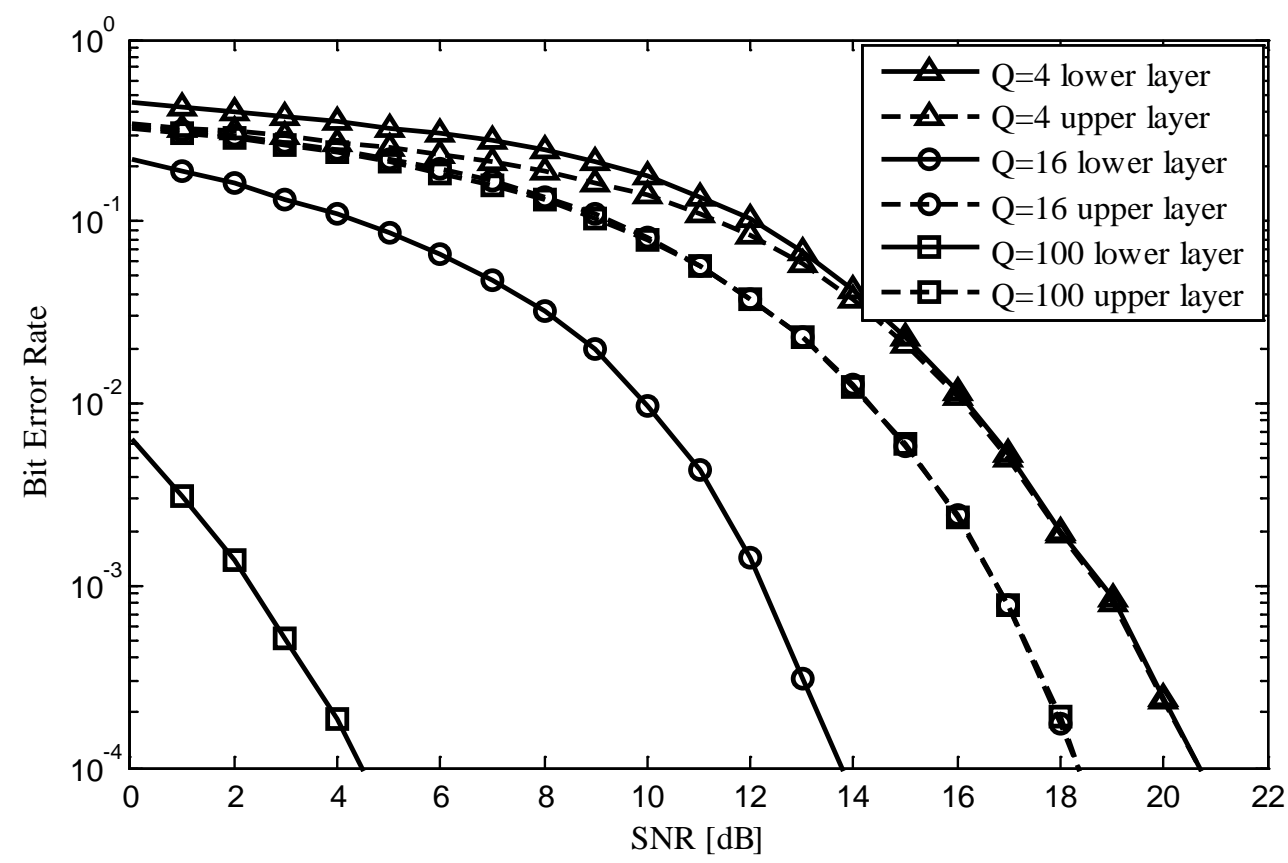

Fig.2 Performance of each layer under different transmitting units

In M-ary modulation system, the overall performance of the system is studied under different transmitting units. With the increase of $Q$, the overall performance of the system significantly improved. We can obtain the performance of each layer under different transmitting units from Fig. 2. It can be seen, along with a substantial increase of $Q$, the detection performance of the upper layer data does not change apparently because the power allocated to this layer is static, whereas the lower layer data detection performance has been improved significantly accompanying high power. It further illustrates that the M-ary modulation is suitable for the system with larger $Q$, or relatively high proportion of the lower layer power.

\section{Conclusion}

In the double-layer OCC system, the lower layer usually occupies higher power but the transmission rate is slower. Aiming at these shortcomings, we have proposed the solution that introducing M-ary modulation to the lower layer signal. The message can be extracted by low-complexity ML detection. Computer simulations have verified the system feasibility. In addition, the general M-ary detection algorithm is extremely significant for the next step to study the multi-layer OCC system.

\section{References}

[1] A.Jovicic, J. Li, T. Richardson, "Visible light communication: opportunities, challenges and the path to market,” IEEE Communications Magazine, 2013, 51(12):26-32.

[2] A. Ashok, M. Gruteser, N. Mandayam, J. Silva, M.Varga, and K. Dana, "Challenge: mobile optical networks through visual MIMO," Proceedings of the sixteenth annual international conference on Mobile computing and networking (Vol.49, pp.105-112). ACM. (2010).

[3] S. Iwasaki, M. Wada, T. Endo, T. Fujii, and M. Tanimoto, "Basic Experiments on Paralle Wireless Optical Communication for ITS,” Intelligent Vehicles Symposium, 2007 IEEE (pp.321-326).

[4] Y. Nakazawa, H. Makino, K. Nishimori, D. Wakatsuki, and H. Komagata, “Indoor positioning using a high-speed, fish-eye lens-equipped camera in Visible Light Communication,” 2013 International Conference on Indoor Positioning and Indoor Navigation (IPIN) (pp.1-8). 
[5] W. Yuan, K. Dana, A. Ashok, M. Gruteser, and N. Mandayam, "Dynamic and invisible messaging for visual MIMO," IEEE Workshop on Applications of Computer Vision (pp.345-352).

[6] A. Ashok, M. Gruteser, N. Mandayam, and K. Dana, "Characterizing multiplexing and diversity in visual MIMO,” Information Sciences and Systems (CISS), 2011 45th Annual Conference on (pp.1-6). IEEE.

[7] S D. Perli, "Pixnet: Designing Interference-free Wireless Links using LCD-Camera Pairs," Massachusetts Institute of Technology, 2011.

[8] S. Kuzdeba, A. M. Wyglinski, and B. Hombs, "Prototype implementation of a visual communication system employing video imagery," Consumer Communications and Networking Conference (CCNC), 2013 IEEE (pp.184-189).

[9] S. Nishimoto, T. Nagura, T. Yamazato, T. Yendo, T. Fujii, and H. Okada, et al. "Overlay Coding for Road-to-Vehicle Visible Light Communication using LED Array and High-Speed Camera,” International IEEE Conference on Intelligent Transportation Systems (Vol.112, pp.1704-1709).

[10] S. Nishimoto, T. Yamazato, H. Okada, and T. Fujii , "High-speed transmission of overlay coding for road-to-vehicle visible light communication using LED array and high-speed camera," Globecom Workshops (GC Wkshps), 2012 IEEE (Vol.16, pp.1234-1238).

[11]H. Y. Zhu, Y. J. Zhu, J. K. Zhang, and Y. Y. Zhang, "A double-layer vlc system with low-complexity ml detection and binary constellation designs," IEEE Communications Letters, 19(4), 561-564.

[12] J. Perez-Ramirez, D. K. Borah, “A single-input multiple-output optical system for mobile communication: modeling and validation,” IEEE Photonics Technology Letters, 26(4), 368-371. 